\title{
Comparison of intra-abdominal abscess formation after laparoscopic and open appendectomy for complicated and uncomplicated appendicitis: a retrospective study
}

\author{
Francesk Mulita ${ }^{1}$, Kerasia-Maria Plachouri' ${ }^{2}$, Elias Liolis ${ }^{3}$, Dimitris Kehagias ${ }^{1}$, Ioannis Kehagias ${ }^{1}$ \\ ${ }^{1}$ Department of Surgery, General University Hospital of Patras, Patras, Greece \\ ${ }^{2}$ Department of Dermatology, General University Hospital of Patras, Patras, Greece \\ ${ }^{3}$ Department of Internal Medicine, Division of Oncology, General University Hospital of Patras, Patras, Greece
}

Videosurgery Miniinv 2021; 16 (3): 560-565 DOI: https://doi.org/10.5114/wiitm.2021.103942

\begin{abstract}
Introduction: Acute appendicitis $(A A)$ is one of the most common causes of acute abdominal pain seen in emergency departments and appendectomy has been the preferred treatment of this disease for decades. Postoperative intra-abdominal abscess (PIAA) complicates 3\% to 25\% of appendectomies and the risk is highest following complicated appendicitis. However, the risk for intra-abdominal abscess formation after appendectomy is still a matter of debate. Aim: To evaluate PIAA formation after open appendectomy (OA) and laparoscopic appendectomy (LA), in particular in complicated appendicitis.

Material and methods: From January 2003 to December 2018, records of patients who underwent appendectomy with diagnosis of appendicitis were retrieved from a computer database for analysis.

Results: During the study period, 1809 appendectomies were performed in our institution (939 LAs and 850 OAs). Twenty conversion cases were recorded. There was no difference between the incidences of PIAA (LA, 3.73\% (35/939) and OA, 3.41\% (29/850); $p>0.05)$. The incidence of PIAA in those with complicated appendicitis was: LA, 11/212 (5.19\%) vs. OA $14 / 198$ (7.07\%); $p>0.05$.

Conclusions: This retrospective study shows that the technique of appendectomy does not appear to affect the incidence of IAAs either in uncomplicated or in complicated appendicitis. However, laparoscopic appendectomy has the advantages of laparoscopic procedures, such as lower hospital stay and earlier return to activities, and should therefore be preferred for acute appendicitis.
\end{abstract}

Key words: appendicitis, appendectomy, complicated appendicitis, intra-abdominal abscess.

\section{Introduction}

Acute appendicitis (AA) is one of the most common causes of acute abdominal pain seen in emergency departments. According to collected data, the annual incidence of acute appendicitis is almost 90 per 100,000 people and the lifetime risk is approximately $8 \%$ [1-3]. It was previously thought that the natural history of appendicitis consisted of inflammation followed by ne- crosis then perforation, but it is now recognized that not all cases progress through this spectrum. Gangrenous or perforated appendicitis occurs in approximately $25 \%$ of cases. It is more likely at the extremes of age, occurring in approximately $40 \%$ of patients under 10 years and $50 \%$ of those over 50 years $[4,5]$.

Appendectomy has been the preferred treatment of appendicitis for decades. Since laparoscop- 
ic appendectomy (LA) was introduced by Semm in 1983, there have been many studies comparing LA versus open appendectomy (OA) for cases with uncomplicated appendicitis and the advantages of the laparoscopic technique have been proved in many published systemic reviews and meta-analyses [6]. However, the role of LA in the management of patients with complicated appendicitis, namely gangrenous, perforated appendicitis and appendiceal abscess, remains controversial. For cases with complicated appendicitis such as gangrenous or perforated appendicitis, laparoscopic appendectomy has replaced open appendectomy in many centers due to decreased blood loss, reduced postoperative pain and hospital stay, fewer overall complications, and an earlier return to usual activities [7].

Post-operative intra-abdominal abscess (PIAA) complicates $3 \%$ to $25 \%$ of appendectomies. The risk is highest following complicated appendicitis. Clinical features include fever, pain, ileus, leucocytosis and an intra-abdominal collection on ultrasound or computed tomography. Management of PIAA includes antibiotics, with or without percutaneous or operative drainage $[8,9]$. PIAA is the most serious complication after appendectomy and, despite its low incidence, is responsible for the majority of reported deaths after LA. Most published studies have reported an increased incidence of PIAA after LA, especially in the treatment of complicated appendicitis [10-12]. However, further analysis is required as there are limited data on the risk of PIAA formation specifically after LA.

\section{Aim}

The purpose of this study was to compare the incidence of PIAA formation in patients who underwent LA or OA for appendicitis, with an emphasis on cases with complicated appendicitis.

\section{Material and methods}

Between January 2003 and December 2018, 1809 patients with suspected diagnosis of AA underwent OA or LA in our institution and were considered for this retrospective study. Informed consent or approval by the local ethics committee was not obtained due to the observational nature of the study. Data were collected from medical and operating theatre records as well as from the hospital-coded database. Patient data were primarily analyzed for the incidence of PIAA. Secondary variables include annual number of appendectomies, patient characteristics (age at operation, gender), length of hospital stay, operative parameters (time and type), presence of complicated appendicitis and need for readmission.

All patients were subjected to clinical examination, blood and urine tests as well as abdominal ultrasound (US) before the operation. Computed tomography (CT) of the abdomen was performed in selected patients to ensure the diagnosis of AA when clinical symptoms or US findings were unclear. Patients undergoing laparoscopic converted to OA or laparotomy were excluded from the study.

Appendectomies (open or laparoscopic) were performed by a team of 2 surgeons. The operative technique was determined by the preference of the consultant who was responsible for the procedure. LAs were performed with a standard 3-trocar technique and an endobag was used for the removal of the specimen from the abdominal cavity.

PIAA was defined as recorded pyrexia and elevated inflammatory markers accompanied by intra-abdominal fluid collection diagnosed radiologically after a minimum of 5 days post-operatively. Complicated appendicitis was diagnosed based on imaging diagnostics and visualization during the operation and was confirmed by histopathology.

\section{Statistical analysis}

Statistical analyses were done using SPSS for Windows 8.0. Student's $t$-test for normally distributed variables, Mann-Whitney $U$ test for skewed variables, $\chi^{2}$ test and Fisher's exact tests were used to compare results between groups. A $p$-value $<0.05$ was considered statistically significant.

\section{Results}

During the 16-year study period, a total of 1809 records of patients operated on for acute appendicitis (AA) in our institution were retrieved from the computer database (Figure 1). Eight hundred fifty appendectomies (47\%) were performed using the open method and 959 (53\%) were performed using the laparoscopic technique. From 2009 LA was performed in the majority of patients. However, in 2016 OA was chosen in the majority of cases, because two new consultant surgeons with a preference for $\mathrm{OA}$ joined our surgical team then. Male patients in both groups outnumbered women. There was no significant difference between the demographic and clin- 
ic-pathological details of patients who underwent $\mathrm{OA}$ and those of patients who underwent LA, except for a lower operative time in the laparoscopic method (47.1 min vs. $57.4 \mathrm{~min} ; p<0.05$ ), as well as lower median hospital stay in the LA $(2.87 \mathrm{~d}$ vs. $3.65 \mathrm{~d}$; $p<0.05)$. Conversation to OA occurred in $20(2.1 \%)$ patients of the LA group. Of the 850 open appendectomies, 198 (23.3\%) were for complicated appendicitis (CA), while 217 CA (22.6\%) were recorded of the 959 laparoscopic procedures $(p>0.05)$. There were
29 (3.4\%) cases of PIAA among 850 patients who underwent OA for AA and 35 (3.7\%) cases of PIAA among 959 patients who underwent LA (Figure 2). There was no significant difference $(p>0.05)$ in the rate of abscess formation between two groups (Table I).

Of the 1809 appendectomies, 415 (22.9\%) were performed for CA. two hundred and seventeen patients underwent LA and 198 underwent OA for CA. Among patients with complicated disease, both groups were comparable in age and gender (Table II).

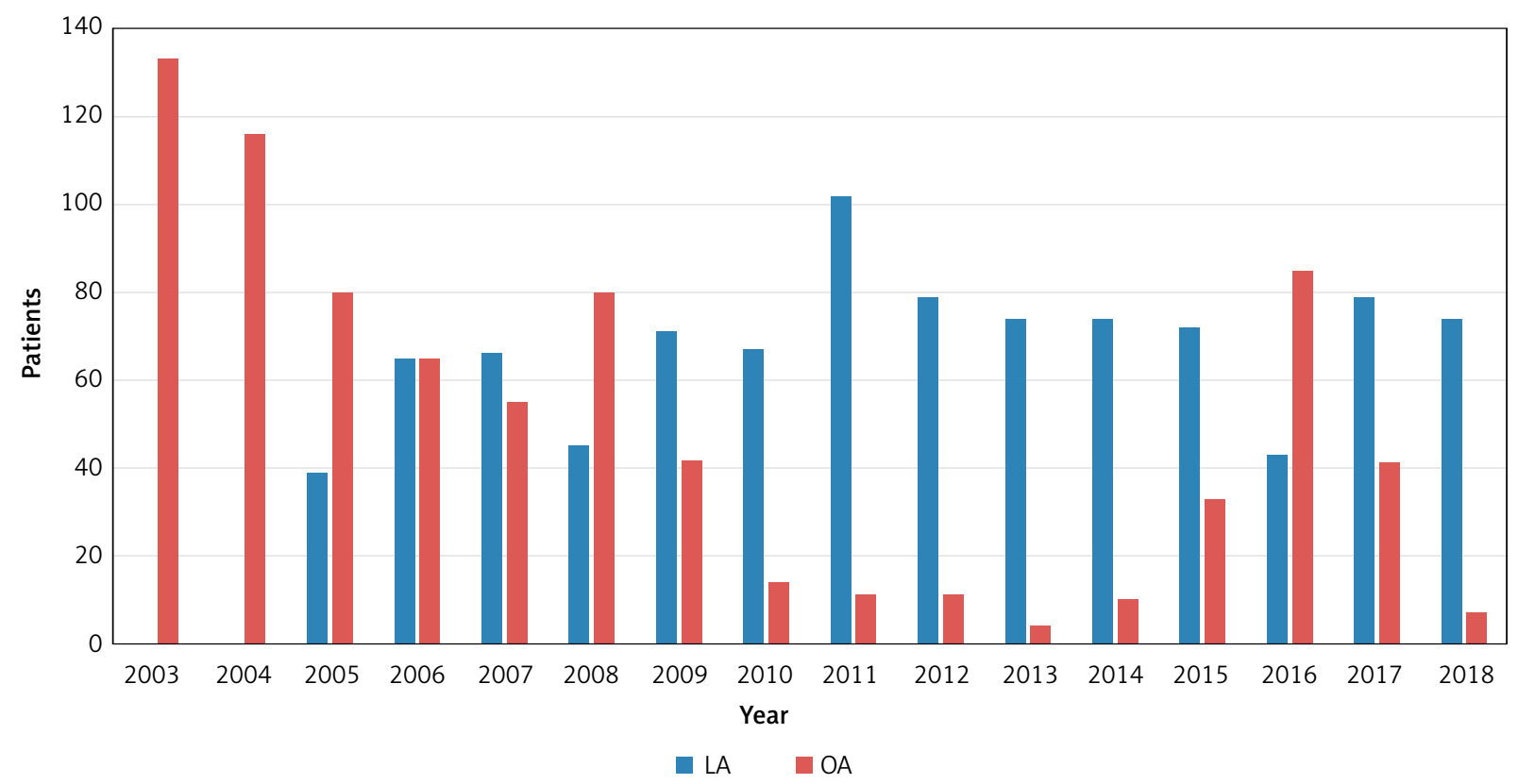

Figure 1. Numbers of laparoscopic appendectomies (LA) and open appendectomies (OA) from 2003 to 2018

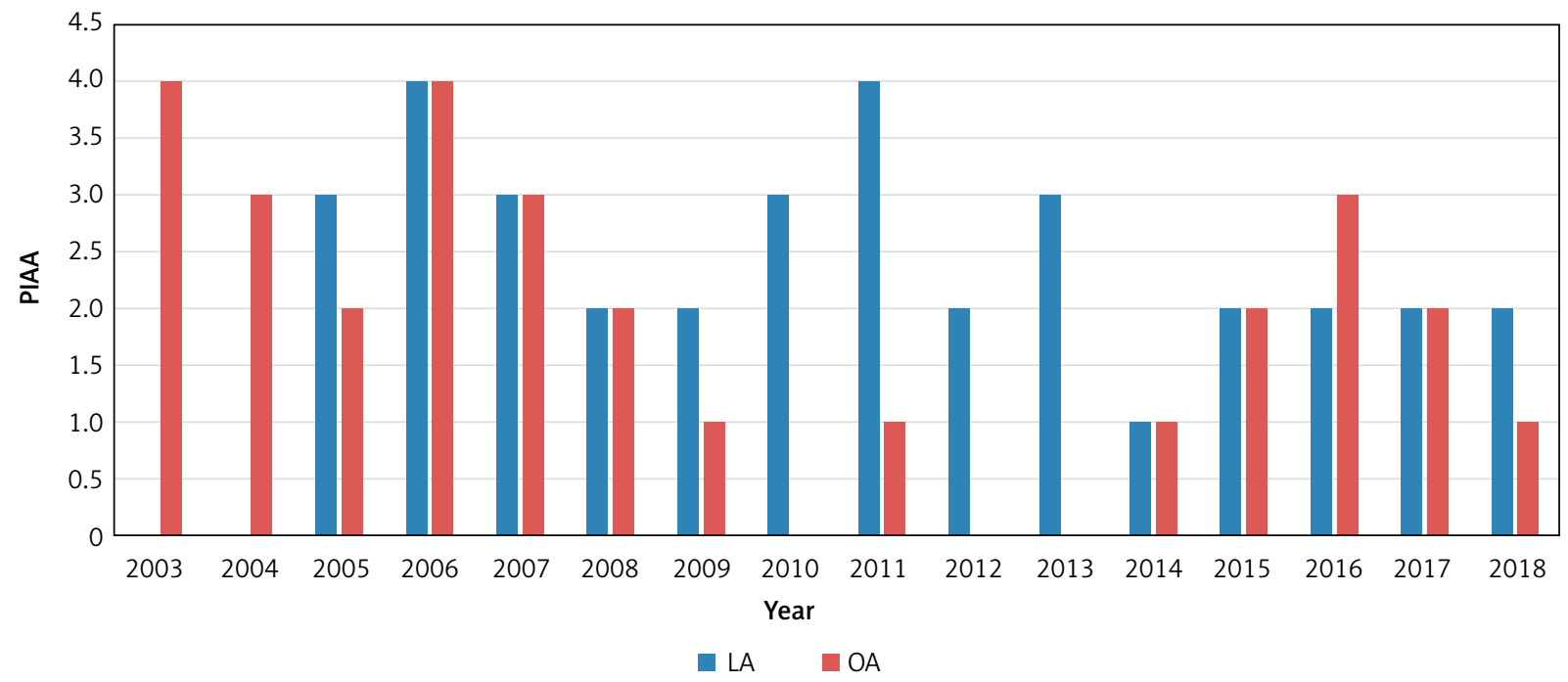

Figure 2. Numbers of postoperative intra-abdominal abscesses (PIAA) during laparoscopic appendectomies (LA) and open appendectomies (OA) from 2003 to 2018 
Table I. Clinico-pathological characteristics of 1809 patients with appendicitis who underwent laparoscopic appendectomies (LA) and open appendectomies (OA) from 2003 to 2018

\begin{tabular}{|lccc|}
\hline Characteristics & OA $(n=850)$ & LA $(n=959)$ & $>$-value \\
\hline Age [years]: & 32.1 & 31.8 & 0.05 \\
\hline Median & $14-85$ & $15-85$ & $>0.05$ \\
\hline Range & & & \\
\hline Sex: & 460 & 528 & $<0.05$ \\
\hline Male & 390 & 431 & $>0.05$ \\
\hline Female & & & $<7.1$ \\
\hline Operative time [min]: & 57.4 & $20-320$ & $<0.05$ \\
\hline Median & $25-280$ & & $>0.05$ \\
\hline Range & 198 & 217 & $<0.05$ \\
\hline Histology: & 652 & 742 & 2.87 \\
\hline Complicated & 3.65 & 35 & 20 \\
\hline Non-complicated & 29 & & \\
\hline Median hospital stay & & & \\
\hline PIAA & & & \\
\hline Conversation rate & & & \\
\hline
\end{tabular}

Median operating time in the LA group (59.2 $\mathrm{min}$ ) was considerably shorter than that in the OA group (65.2 $\mathrm{min})$. In addition, median lengths of hospital stay were 5.91 in the OA group and 5.04 in the LA group $(p<0.05)$. In $5(2.3 \%)$ patients of the LA group, conversion to an open procedure occurred because of technical difficulties (Figure 2).

A PIAA developed in 14 out of 198 patients who underwent $\mathrm{OA}$ for $\mathrm{CA}$, while abscess formation was recorded in 11 out of 212 patients with CA of the LA group (Figure 3). There was no significant difference $(p>0.05)$ in the rate of abscess formation between two groups with complicated disease.

\section{Discussion}

There has been persistent concern over an apparent increase in the incidence of intra- abdominal

Table II. Clinico-pathological characteristics of 415 patients with complicated appendicitis who underwent laparoscopic appendectomies (LA) and open appendectomies (OA) from 2003 to 2018

\begin{tabular}{|c|c|c|c|}
\hline Characteristics & OA $(n=198)$ & LA $(n=217)$ & $P$-value \\
\hline Age [years]: & & & $>0.05$ \\
\hline Median & 31.9 & 32.3 & \\
\hline Range & $17-85$ & $16-85$ & \\
\hline Sex: & & & $>0.05$ \\
\hline Male & 111 & 124 & \\
\hline Female & 87 & 93 & \\
\hline \multicolumn{4}{|l|}{ Operative time [min]: } \\
\hline Median & 65.2 & 59.2 & $<0.05$ \\
\hline Range & $40-280$ & $40-320$ & \\
\hline Median hospital stay & 5.91 & 5.04 & $<0.05$ \\
\hline PIAA & 14 & 11 & $>0.05$ \\
\hline Conversation rate & & 5 & $<0.05$ \\
\hline
\end{tabular}




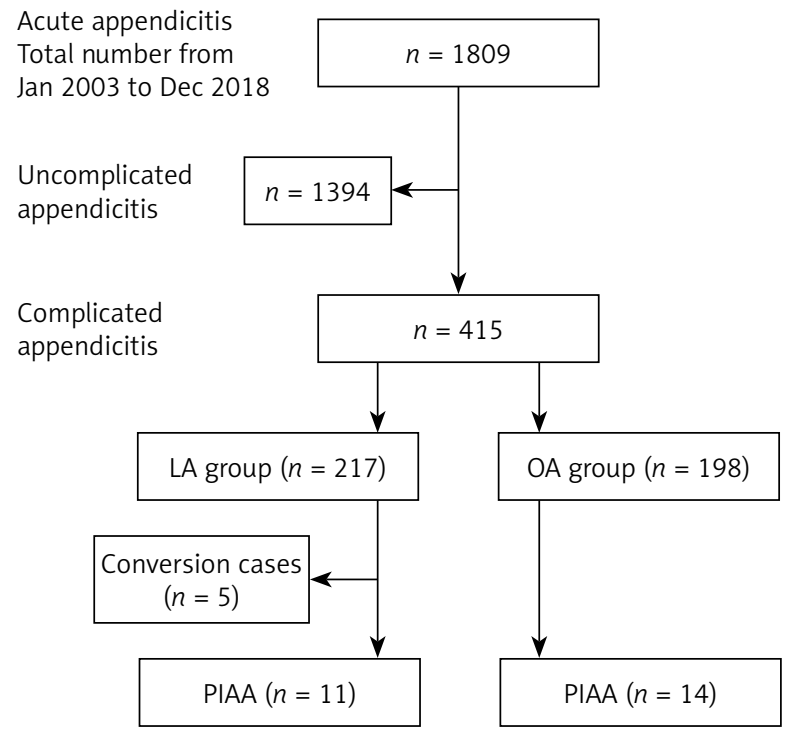

Figure 3. Flowchart of 1809 patients who underwent laparoscopic appendectomies (LA) and open appendectomies (OA) from 2003 to 2018 and number of cases of post-operative intra-abdominal abscess

abscess after laparoscopic appendectomy [13, 14]. However, the present study demonstrated that the risk for PIAA formation after laparoscopic appendectomy is not larger than after the open technique, even in cases with complicated appendicitis.

Sauerland et al. evaluated 67 studies comparing $L A$ versus $O A$ for suspected appendicitis in a Cochrane review and an increase risk of PIAA was reported in $\mathrm{LA}(\mathrm{OR}=1.87 ; 95 \% \mathrm{Cl}: 1.9-2.93)$ [14]. $A$ retrospective cohort study performed by Azarias et al., included 1924 adults who underwent LA and 540 who underwent OA in two centers from 19962007. This study showed no significant difference in the incidence of PIAA between the two groups $(2.2 \%$ vs. $1.9 \%, p>0.05)$ or in patients with complicated appendicitis (5.9\% vs. $4.1 \%, p>0.05$ ) [7].

Based on our study PIAA developed in $25(6 \%)$ patients with complicated appendicitis and 39 (2.8\%) patients with non-complicated appendicitis. According to the literature, the presence of complicated appendicitis is the only independent risk factor for PIAA formation [15]. Post-operative complications such as PIAA may also depend on the skills of the surgeon, as demonstrated by Katkhouda et al., who introduced a specialized laparoscopic team for LA in their institution and there was a decrease in the PIAA rate from $2.4 \%$ to $0.4 \%$ [12].
In our study, we found that the operative time as well as the hospital stay of patients in the LA group was significantly shorter than the OA group. Similar finding were noted in patients with complicated appendicitis. Some studies reported a shorter and some a longer operative time for laparoscopic than for open appendectomy. This heterogeneity might be explained by different laparoscopic skill levels in the surgeons of each center [16]. The majority of surgeons of our department have skills in advanced laparoscopic surgery. It has been reported in many studies that the length of stay is shorter in patients who underwent LA. Furthermore, patients who underwent LA have lower incidence of wound infection $[6,17]$.

Our study has several limitations because patients were assessed retrospectively from a single center. In addition, data about preoperative and post-operative antibiotics have not been recorded. According to the literature, preoperative antibiotics administered to patients with appendicitis have shown great efficacy in reducing complication rates. However, there is a lot of controversy about their benefit in the post-operative period [15]. Moreover, cases with surgical drains were not recorded and the correlation between surgical drains and PIAA formation was not evaluated in the present study. The use of a surgical drain on a routine basis postoperatively has not been proven to improve outcomes and the exact impact on risk of PIAA in complicated appendicitis remains doubtable. However, a study by Alleman et al. reported that the use of a surgical drain increases the risk of PIAA formation [18]. Other post-operative complications such as wound infections were not recorded in the present study.

Our study indicates the need for a prospective randomized controlled trial of OA vs LA that specifically addresses complicated appendicitis.

\section{Conclusions}

This retrospective study shows that the technique of appendectomy does not appear to affect the incidence of IAAs either in non-complicated or in complicated appendicitis. However, laparoscopic appendectomy has the advantages of laparoscopic procedures, such as lower hospital stay and earlier return to activities and should therefore be preferred for acute appendicitis. 


\section{Conflict of interest}

The authors declare no conflict of interest.

\section{References}

1. Sauerland S, Jaschinski T, Neugebauer EA. Laparoscopic versus open surgery for suspected appendicitis. Cochrane Database Syst Rev 2010; 10: CD001546.

2. Jastrzębski M, Krasnodębski M, Szczęśniak M, et al. Serum bilirubin concentration as a marker of severity of acute appendicitis. Videosurgery Miniinv 2020; 15: 117-22.

3. Mulita F, Oikonomou N, Provatidis A, et al. Roseomonas gilardii in patient with leukemia and acute appendicitis: case report and review. Pan Afr Med J 2020; 36: 283.

4. Bhangu A, Soreide K, Di Saverio S, et al. Acute appendicitis: modern understanding of pathogenesis, diagnosis, and management. Lancet 2015; 386: 1278-87.

5. Buckius MT, McGrath B, Monk J, et al. Changing epidemiology of acute appendicitis in the United States: study period 1993 2008. J Surg Res 2012; 175: 185-90.

6. Yau KK, Siu WT, Tang CN, et al. Laparoscopic versus open ap pendectomy for complicated appendicitis. J Am Coll Surg 2007; 205: 60-5.

7. Asarias JR, Schlussel AT, Cafasso DE, et al. Incidence of postop erative intraabdominal abscesses in open versus laparoscopic appendectomies. Surg Endosc 2011; 25: 2678-83.

8. Fike FB, Mortellaro VE, Juang D, et al. The impact of postoperative abscess formation in perforated appendicitis. J Surg Res 2011; 170: 24-6.

9. Gupta R, Sample C, Bamehriz F, et al. Infectious complications following laparoscopic appendectomy. Can J Surg 2006; 49: 397-400.

10. Martin LC, Puente I, Sosa J, et al. Open versus laparoscopic appendectomy: a prospective randomized comparison. Ann Surg 1995; 222: 256-62.

11. Paik P, Towson BA, Anthone GJ, et al. Intra-abdominal abscesses following laparoscopic and open appendectomies. J Gastrointest Surg 1997; 1: 188-93.

12. Katkhouda N, Friedlander MH, Grant SW, et al. Intraabdominal abscess rate after laparoscopic appendectomy. Am J Surg 2000; 180: 456-61.

13. Nataraja RM, Teague WJ, Galea J, et al. Comparison of intraabdominal abscess formation after laparoscopic and open appendicectomies in children. J Pediatr Surg 2012; 47: 317-21.

14. Sauerland S, Jaschinski T, Neugebauer EA. Laparoscopic versus open surgery for suspected appendicitis. Cochrane Database Syst Rev 2010; 10: CD001546.

15. Lasek A, Pędziwiatr M, Wysocki M, et al. Risk factors for intraabdominal abscess formation after laparoscopic appendectomy - results from the Pol-LA (Polish Laparoscopic Appendectomy) multicenter large cohort study. Videosuregry Miniinv 2019; 14: 70-8.

16. Horvath P, Lange J, Bachmann R, et al. Comparison of clinical outcome of laparoscopic versus open appendectomy for complicated appendicitis. Surg Endosc 2017; 31: 199-205.
17. Richard KF, Fisher KS, Flores JH, et al. Laparoscopic appendectomy: comparison with open appendectomy in 720 patients. Surg Laparosc Endosc 1996; 6: 205-9.

18. Allemann P, Probst $H$, Demartines $N$, et al. Prevention of infectious complications after laparoscopic appendectomy for complicated acute appendicitis--the role of routine abdominal drainage. Langenbecks Arch Surg 2011; 396: 63-8.

Received: 6.10.2020, accepted: 6.01.2021 